\title{
Wavelet Domain Bilateral Filtering with Subband Mixing for Magnetic Resonance Image Enhancement
}

\author{
Zeinab A. Mustafa*, Banazier A. Abrahim, Inas A. Yassine, \\ Nourhan Zayed, and Yasser M. Kadah \\ Biomedical Engineering Department, Cairo University, Giza 12613, Egypt
}

\begin{abstract}
A method for magnetic resonance image denoising based on wavelet domain bilateral filtering (WDBF) is proposed. The main problem in bilateral filtering based methods is that the choice of filtration parameters has a trade-off between preserving edges and noise removal. In this work, a solution that would allow different components of the image to be filtered using different parameters is presented. The bilateral filtering is applied in a customized manner to different wavelet subbands and followed by subband mixing to form the final image. The proposed method is implemented to filter magnetic resonance images and verified both qualitatively and quantitatively. Verification of the new method was carried out on synthetic as well as real data sets. Qualitative and quantitative comparisons with present techniques indicate that the proposed method produces superior denoising results and suggesting potential for clinical application to boost the signal-to-noise ratio of low magnetic field scanners.
\end{abstract}

Keywords: Magnetic Resonance Imaging, Denoising Algorithms, Multiresolution Processing, Method Noise, Wavelet Transform, Bilateral Filter, Image Quality Factor, Gaussian Noise.

\section{INTRODUCTION}

In magnetic resonance imaging (MRI), the signal-to-noise ratio (SNR) depends mainly on the strength of the static magnetic field of the system in addition to the protocol used in image acquisition. For example, in lower field MRI systems such as open MRI, the SNR is very low and it is a common practice to repeat the image acquisition multiple times to do averaging in order to improve the image quality. Also, in some protocols such as with diffusion tensor imaging the SNR is usually very low and again multiple scans and averaging are done. This amounts to added discomfort to the patient in addition to lower efficiency of MRI facilities. Besides improving visual quality of the images required for correct diagnosis, image denoising is usually used as a preprocessing step to improve the accuracy of subsequent image processing algorithms such as registration or segmentation. As a result, many denoising methods have been developed by many groups such as wavelet shrinkage and anisotropic diffusion based method. ${ }^{1,2}$ An important class of denoising methods is based on the bilateral filter $^{3}$ which computes a weighted sum of the pixels in a local neighborhood based on both the spatial

${ }^{*}$ Author to whom correspondence should be addressed. separation and the difference in intensity. The filtered image can be calculated as follows, ${ }^{3}$

$$
(X)=\breve{I} \frac{1}{C} \sum_{y \in N(x)} e^{\left(-|y-x|^{2} / 2 \sigma_{d}^{2}\right)} e^{\left(-|I(y)-I(x)|^{2} / 2 \sigma_{r}^{2}\right)} I(y)
$$

where $\sigma_{d}$ and $\sigma_{r}$ are parameters controlling the filter support along both spatial and intensity variables, respectively, $N(x)$ is a spatial neighborhood of $I(x)$, and $C$ is a normalization constant.

Although the bilateral filter was originally proposed as an independent idea, recent papers have pointed out theoretical connections with several other techniques. For example, it was shown to be related to the Jacobi algorithm, ${ }^{4}$ anisotropic diffusion, ${ }^{5,6}$ and nonlocal means filter with patch size of one pixel. ${ }^{6}$ Hence, the bilateral filtering idea offers generality and can be tailored to custom applications. The main problem is that the choice of bilateral filtration parameters has a trade-off between preserving edges and noise removal as with many methods. In this work, we propose a solution that would allow different components of the image to be filtered using different bilateral filtering parameters and hence allow for better performance. The proposed method is implemented to filter magnetic resonance images and verified both qualitatively and quantitatively. 


\section{NOISE PROPERTIES OF MR DATA}

The raw MRI data consists of complex valued samples with real and imaginary parts that can each be modeled as the summation of deterministic and Gaussian random noise components. ${ }^{7}$ After reconstruction by inverse Fourier transformation, the real and imaginary data are still corrupted with Gaussian white noise because of the orthogonality of the Fourier transform. However, it is common practice to transform the complex valued images into magnitude and phase images. ${ }^{7-8}$ The magnitude image is formed by calculating the magnitude, pixel by pixel, from the real and the imaginary images. This is a nonlinear mapping and therefore the noise distribution is no longer Gaussian and the noise within each pixel belongs to the Rician distribution. ${ }^{9-10}$ This distribution form depends in a complex manner on the value of the means of the real and imaginary part distributions, which are unknown (in fact their values represent the solution for the denoising problem). The Rician distribution is far from being Gaussian for small $\operatorname{SNR}(A / \sigma \leq 1)$. For ratios as small as $A / \sigma=3$, however, it starts to approximate the Gaussian distribution. A special case of the Rician distribution is obtained in image regions where only noise is present, i.e., $A=0$, which reduces to the known the Rayleigh distribution. Since practical acquisitions of tissue signals involve $A / \sigma$ that is greater than 3 , it is possible to use the Gaussian white noise as a good model for the type of noise encountered in the MRI magnitude images.

\section{METHODOLOGY}

\subsection{Wavelet Domain Bilateral Filter}

Image features usually have different spatial details spanning particular spatial frequency bands, whereas noise is usually present everywhere in a very much uniform manner. Therefore, multiresolution analysis can be useful since it is possible to differentiate noise from actual image information in some resolution levels more than others. ${ }^{11}$ We observe that this can be used to tailor the filtering parameters in different resolution levels. Therefore, we propose using the bilateral filter in a multiresolution manner summarized as follows:

(a) Processing the original image $I$ using two optimal sets of bilateral filtering parameters: one is optimized for noise components removal while the other is optimized for image feature preservation. This yields two images $I_{o}$ (low noise and poor feature preservation) and $I_{u}$ (medium noise and excellent feature preservation) similar to Ref. [11].

(b) Decomposing both $I_{o}$ and $I_{u}$ into four subbands using Daubachies-8 filters (low- and high-frequency subbands).

(c) Mixing the high frequency subbands of $I_{o}$ and the low frequency subbands of $I_{u}$ to produce the filtered wavelet transformation.

(d) Reconstruction of the final image is done using inverse wavelet transformation from the combination of the selected subbands.

A block diagram of the proposed method is presented in Figure 1.

\subsubsection{Wavelet Decomposition}

Wavelet decomposition is performed on both $I_{o}$ and $I_{u}$ images using a particular wavelet family of choice (here, Daubachies-8

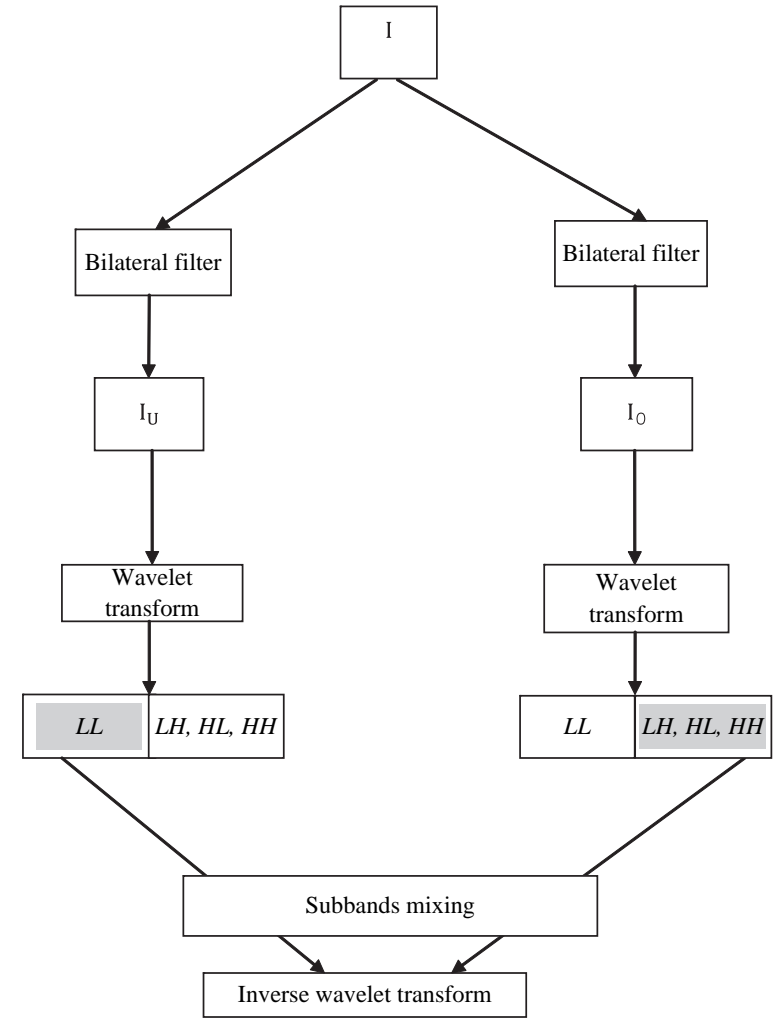

Fig. 1. A block diagram of the proposed method. First, the noisy image $I$ is denoised with two sets of filtering parameters $I_{u}$ and $I_{o}$. Then, $I_{u}$ and $I_{o}$ are decomposed into low- and high-frequency subbands by wavelet transform (WT). The lowest frequency of $I_{u}$ are mixed with the three highest-frequency subbands of $I_{o}$ (i.e., $\mathrm{HL}, \mathrm{LH}$, and $\mathrm{HH}$ ). Finally, the resultant image is obtained by inverse WT.

family was used). For each image, four subbands are obtained: LL, LH, HL, and HH. In the four wavelet subbands obtained with $I_{o}$, all frequencies of noise were removed but tend to blur some features in the original image. In the four wavelet subbands obtained with $I_{u}$, they generally preserve image features but at the expense of poor noise removal.

\subsubsection{Investigation of Optimal Parameter Selection}

The behavior of the bilateral filter ${ }^{3}$ relies on the selection of $\sigma_{d}$ and $\sigma_{r}$ in Eq. (1) that control the extent of spatial and intensity domain filtration. The issue of choosing optimal parameter values is not been resolved from a theoretical perspective. Also, empirical studies available in the literature focus on different image types (cf. Ref. [11]). Hence, it is necessary to have our own empirical study of parameter selection to obtain the optimal parameters to be used in this study.

\subsection{Evaluation Criteria of Filtering Results}

As defined in Ref. [3], let $u$ be the original image and $D_{h}$ a denoising operator depending on $h$. Then the method noise of $u$ is defined as the image difference,

$$
\left(D_{h}, u\right)=u-D_{h}(u)
$$

This method noise should be as similar to Gaussian white noise as possible if the denoising operator preserves the image features. 
Four criteria can and will be taken into account in the comparison of denoising methods:

(a) A display of typical artifacts in denoised images.

(b) A formal computation of the method noise on smooth images, evaluating how small it is in accordance with image local smoothness.

(c) A comparative display of the method noise of each method on MR images with five different levels of noise.

(d) A classical comparison based on noise simulation: it consists of taking a good quality image, adding Gaussian noise with known $\sigma$, and then computing the best image recovered from the noisy one by each method.

\subsection{Image Quality Evaluation Metrics}

To quantify the performance, the noise reduction method, various measures may be used. The commonly preferred measures are mean squared error (MSE), and root mean squared error (RMSE), signal to noise ratio (SNR), peak signal to noise ratio (PSNR), ${ }^{12}$ which can be evaluated as a function of the original, $g_{i, j}$, and the denoised, $f_{i, j}$, the metrics used in our study are defined as follows:

(a) The root-mean-square error (RMSE), which is the square root of the squared error averaged over an $M \times N$ window: ${ }^{13}$

$$
R M S E=\sqrt{\frac{1}{M N} \sum_{i=1}^{M} \sum_{j=1}^{N}\left(g_{i, j}-f_{i, j}\right)^{2}}
$$

(b) The signal-to-noise ratio (SNR) is given by: ${ }^{14}$

$$
S N R=10 \log _{10} \frac{\sum_{i=1}^{M} \sum_{j=1}^{N}\left(g_{i, j}^{2}+f_{i, j}^{2}\right)}{\sum_{i=1}^{M} \sum_{j=1}^{n}\left(g_{i, j}-f_{i, j}\right)^{2}}
$$

(c) The peak SNR (PSNR) is computed using: ${ }^{14}$

$$
P S N R=-10 \log _{10} \frac{\mathrm{MSN}}{g_{\max }^{2}}
$$

where $g_{\max }^{2}$ is the maximum intensity in the unfiltered image. The PSNR is higher for a better-transformed image and lower for a poorly transformed image. It measures image fidelity, which is how closely the denoised image resembles the original image.

\section{EXPERIMENTAL RESULTS AND DISCUSSION}

To experimentally verify the new method, more than twenty real brain MR images of different orientations were used from different sources (e.g. Refs. [17, 18], in addition to data collected from Biomedical Imaging Technology Center). In our quantitative experiments, images collected using averaging were corrupted with Gaussian white noise of five different levels. This provides accurate simulation since the Rician noise in brain tissues can be generally well approximated by Gaussian white noise. Figure 2 shows two such images before and after corruption with noise and their method noise. All experiments were performed using MATLAB 7.0 (Mathworks Inc.) on a Pentium Core-2 Duo Laptop with 2 GB of RAM.

To understand the relationship between $\sigma_{d}$ and $\sigma_{r}$, the following experiments were done. Gaussian white noise was added to a reference image and the bilateral filter was applied for different values of the parameters $\sigma_{d}$ and $\sigma_{r}$. Figure 3 illustrates the results for different combinations of parameter values. As the range parameter $\sigma_{r}$ increases, the bilateral filter is nearly constant over the intensity interval of the image. Increasing the spatial
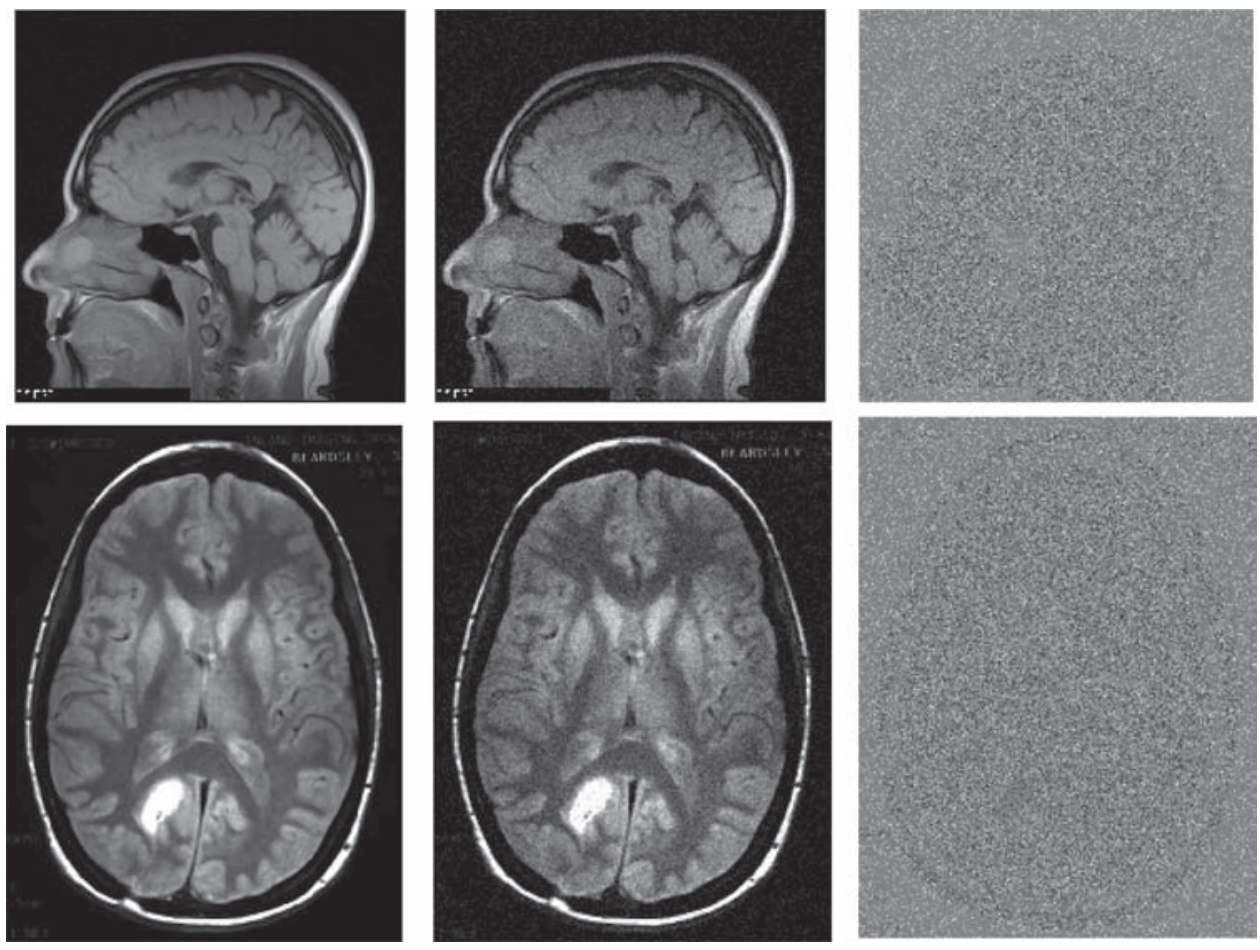

Fig. 2. From left to right: $T 1$-weighted noise-free MR image, the corresponding noisy image $(\sigma=5 \%)$ and applied the method noise (difference between both images). 
$\sigma_{\mathrm{d} /} \sigma_{\mathrm{r}}$

0.01
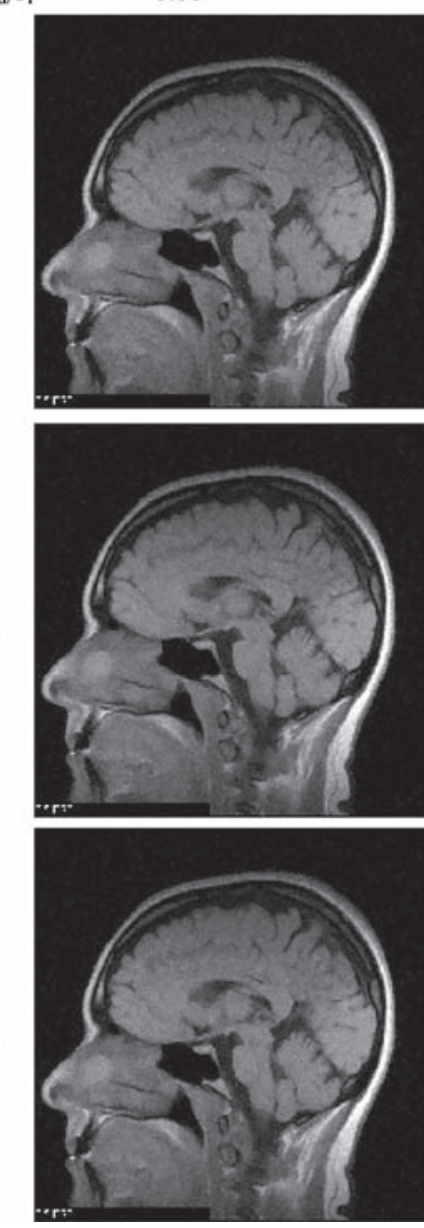

0.05
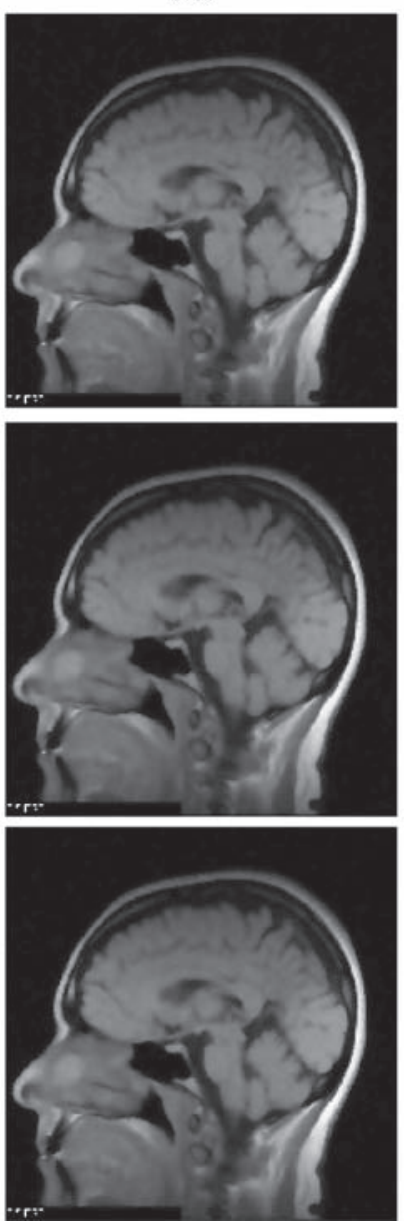

0.2
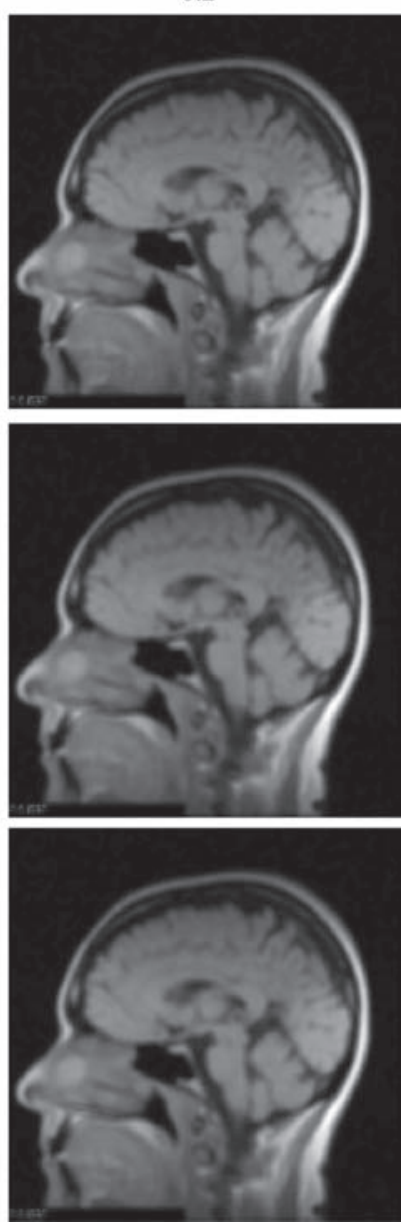

Fig. 3. The bilateral filter range and spatial parameters provide more versatile control than other methods. As soon as either of the bilateral filter weights reaches values near zero, no smoothing occurs. As a consequence, increasing the spatial sigma will not blur an edge as long as the range sigma is smaller than the edge amplitude.
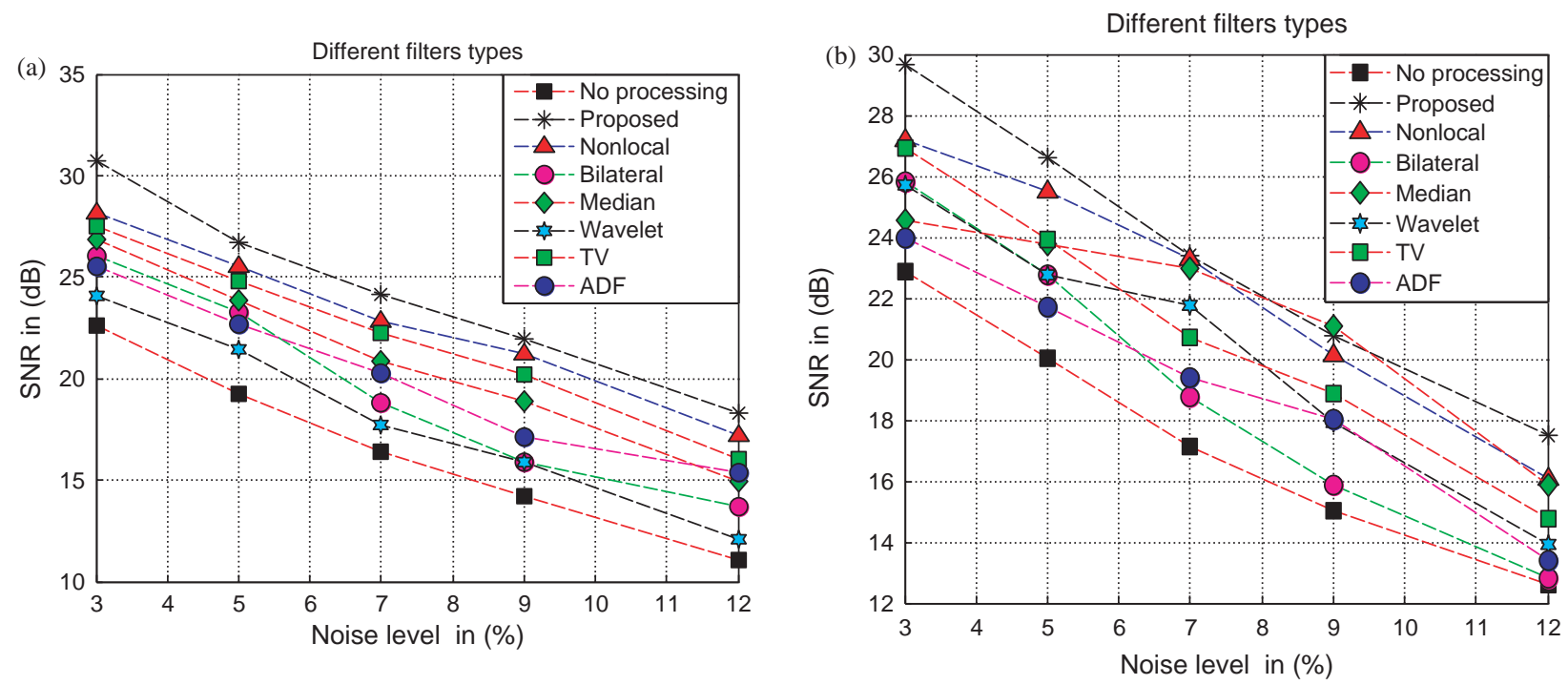

Fig. 4. Comparison of the different denoising on two different classes of images. 


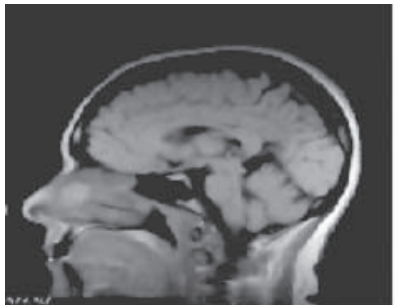

(a) LL subband

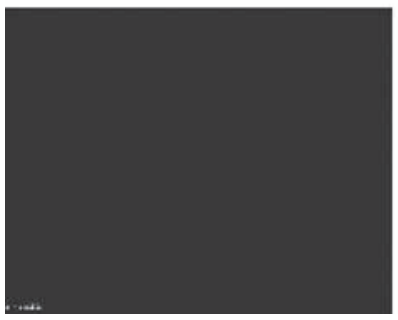

(c) LH subband

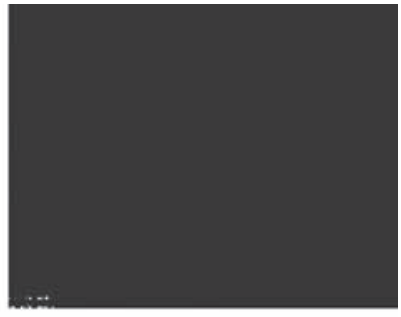

(b) HL subband

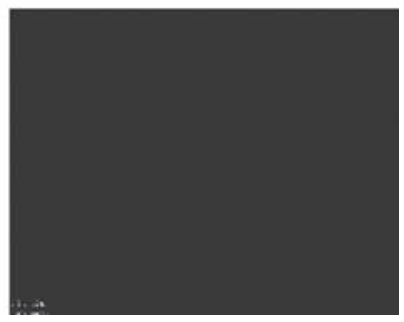

(d) $\mathrm{HH}$ subband
Fig. 5. Four subbands obtained by wavelet decomposition adapted to the noise components removal (with $\sigma_{d}=8$ and $\sigma_{r}=0.2$ ).

parameter $\sigma_{d}$ smooth larger features. In practice, adapting the range parameter $\sigma_{r}$ to estimates of the local noise level yields better results. An important characteristic of bilateral filtering is that the weights are multiplied and so, if either of the weights is close to zero, no smoothing occurs.

The accuracy of the proposed method and compared filters over usual noise levels $(3 \%, 5 \%, 7 \%, 9 \%$, and $12 \%$ of the maximum $T 1$-weighted image intensity) can be seen in Figure 4 . As presented in Figure 4 our proposed WDBF produced the best SNR value whatever the noise level is. The SNR values between the noisy image and original image is called "No processing" and is used as reference. Figures 5 and 6 show the wavelet subbands LL, $\mathrm{HL}, \mathrm{LH}$ and $\mathrm{HH}$ obtained by wavelet decomposition after denoising the original image $I$ using two sets of filtering parameters: one optimized for noise removal (i.e., $\sigma_{d}=8$ and $\sigma_{r}=0.2$ ) and

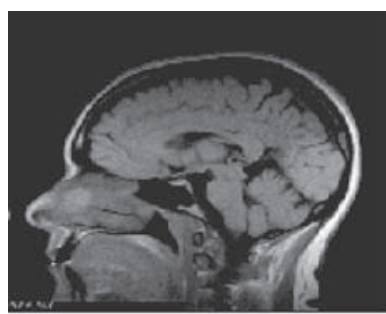

(a) LL subband

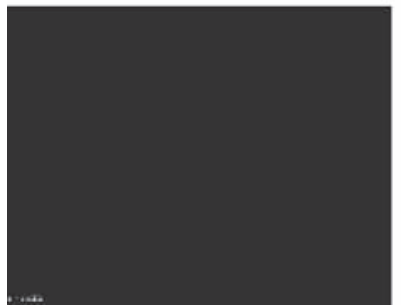

(c) LH subband

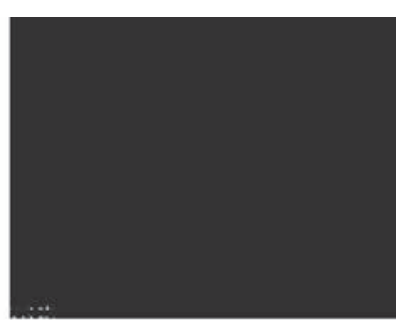

(b) HL subband

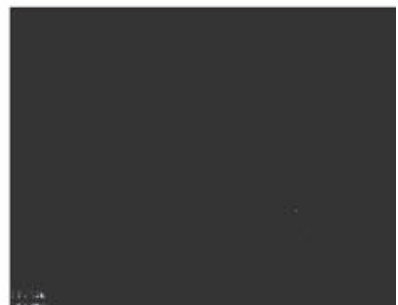

(d) HH subband
Fig. 6. Four subbands obtained by wavelet decomposition adapted to the image features preservation (with $\sigma_{d}=2$ and $\sigma_{r}=0.05$ ). the other optimized for image feature preservation (i.e., $\sigma_{d}=2$ and $\sigma_{r}=0.05$ ) respectively. Part (a) in both figures shows the low frequency subband LL, whereas parts (b), (c) and (d) present the high frequency subbands of horizontal, vertical and diagonal directions, respectively. In Figure 5, one can see that most of the texture concentrates on the three high frequency subbands. In Figure 6(c), the noise can be visualized but the texture is overwhelmed by noise.

Qualitative and quantitative comparisons were done in this study to evaluate the effectiveness of the new method. The performance of the new method was compared against several well-established (and widely used) filtering algorithms; namely the anisotropic diffusion filter (ADF),${ }^{15}$ Median, total variation (TV), ${ }^{16}$ nonlocal mean (NLM), wavelet thresholding, and bilateral filtering. The results of this comparison are shown in Figures 7 and 8 for two sample transverse and sagittal slices of the head. The visual comparison shows the advantage of the proposed filter to increase the apparent SNR of the MR images without noticeably affecting image structures. The results presented in these figures were obtained for a $5 \%$ of Gaussian noise, but this screening was performed for the five levels of noise with similar results.

\subsection{Method Noise Comparison}

As described above, the method noise is used to measure the geometrical features or details that are not preserved by the denoising process and which are eliminated. From Figures 9 and 10, one can observe that the method noise of the anisotropic filter shows highlighting of the edges and high frequency features, but effective within texture. Also, the method noise of the total variation indicates modification of most structures and details of the image and that of the nonlocal mean method shows reduction in the loss of details, and removed noise. The method noise of the wavelet Thresholding indicates poorer performance in removing noise and also shows artifacts in the denoised image. The results of bilateral Filtering shows smoothed images while preserving edges. The median filtering shows unnatural edge enhancement and small edges blurring. On the other hand, the method noise of the proposed wavelet domain bilateral filtering was clearly clean from image edges and looks more like Gaussian white noise than any of the other methods.

To quantitatively verify the quality of the filters, Tables I and II show the quantitative performance measures including SNR, Peak SNR, MSE, and RMSE for the different types of noise reduction filters. These results were obtained using Monte Carlo simulations of adding Gaussian random noise to real MRI images obtained with averaging using different noise levels. A total of 10 simulations for 10 images were used. Best values were obtained for the proposed method, nonlocal mean, median, and total variation, with lower MSE, and RMSE, and higher SNR, and PSNR. The proposed algorithm was quantitatively compared with the other six referred methods showing a lower MSE and RMSE and higher SNR and PSNR in almost all the cases as demonstrated in Figures 11 and 12.

It should be noted that the proposed method is a modified denoising method based on the method by Tomasi et al. ${ }^{3}$ where it is enhanced using an automatic tuning of the filtering parameter, and a mixing of wavelet subbands to overcome the main limitation of the classical bilateral filter. It also bears resemblance to the multiresolution bilateral filter proposed in Ref. [14] for 


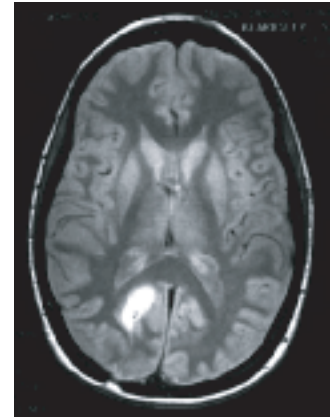

(a)

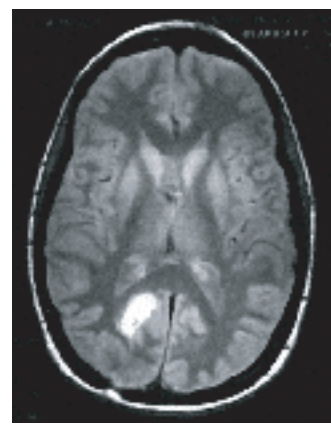

(e)

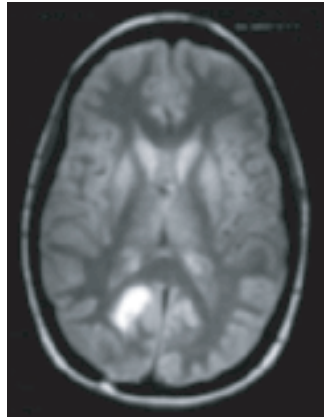

(b)

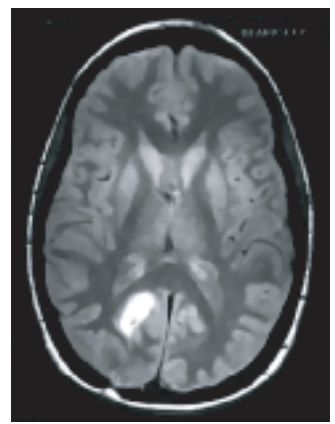

(f)

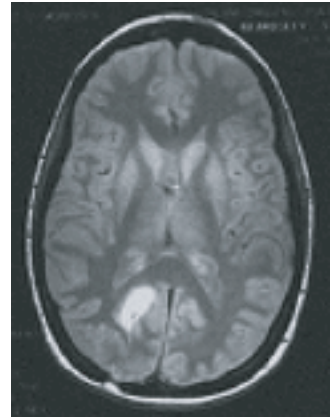

(c)

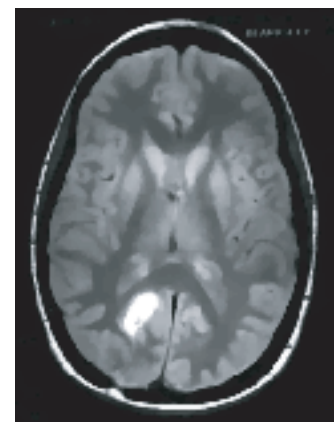

(g)

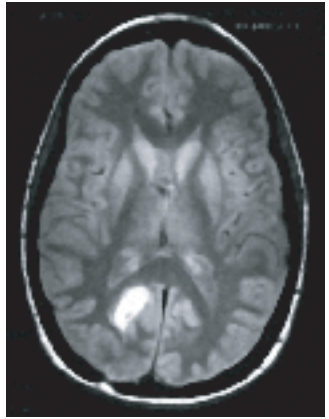

(d)

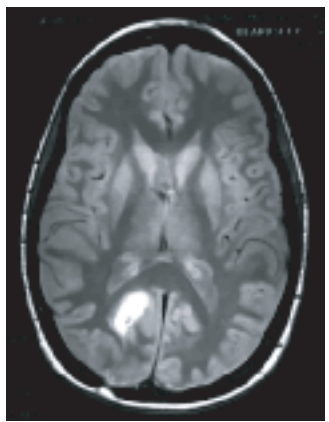

(h)

Fig. 7. Qualitative comparison of the filtering results obtained with the different compared methods. The original image given in (a) obtained via the compared methods given in (b)-(k). (a) Original image, (b) ADF, (c) wavelet, (d) median, (e) bilateral filters, (f) NL-mean, (g) TV, and (h) proposed method.

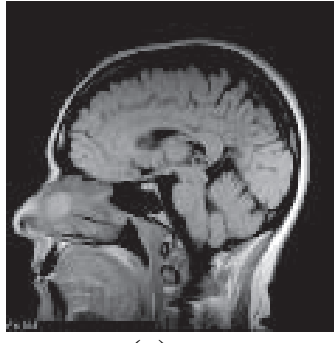

(a)

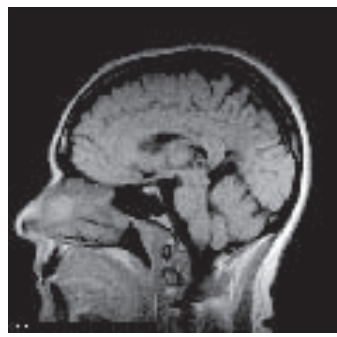

(e)

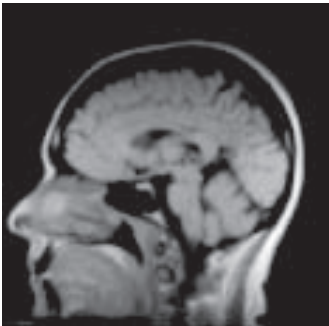

(b)

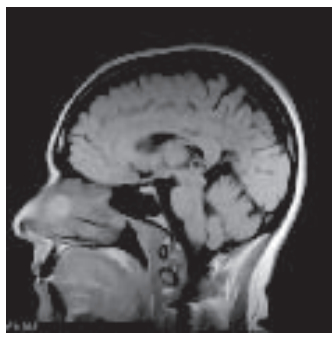

(f)

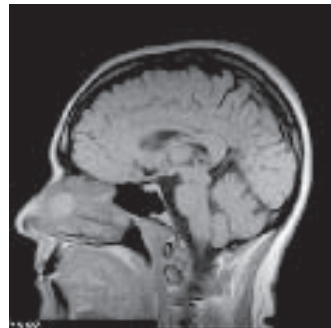

(c)

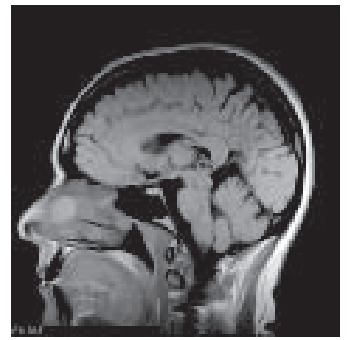

(g)

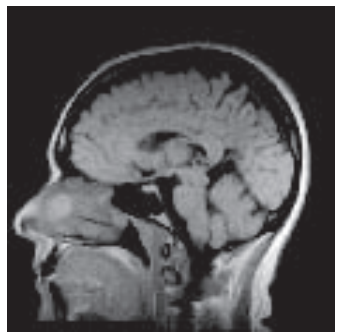

(d)

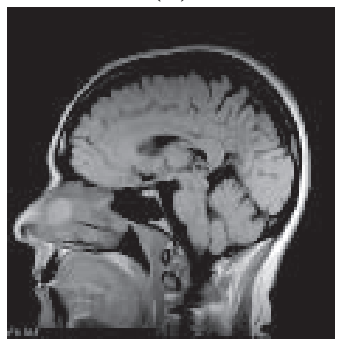

(h)

Fig. 8. Qualitative comparison of the filtering results obtained with the different compared methods. The original image given in (a) obtained via the compared methods given in (b)-(k). (a) Original image, (b) ADF, (c) wavelet, (d) median, (e) bilateral filter, (f) NL-mean, (g) TV, and (h) proposed method.

different image type with a different strategy in using double bilateral filtering and subband mixing similar to what was proposed in Ref. [19] for a different processing chain.

The issue of selecting which wavelet family to use in different problems is not resolved in image processing in general. Here, our experiments did not show fundamental differences between different wavelet families to suggest that one of them is better.
So, the wavelet family used here can be considered as a specification to "one" working version of the method so that there are no missing steps for complete implementation and also for the reader to be able to reproduce the results to the ones here without confusion. We encourage further research on the problem of optimizing the selection of wavelets or even the design of custom ones for different problems. 


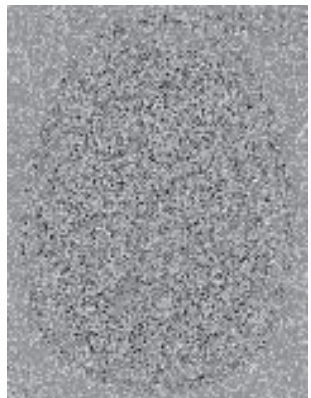

(a)

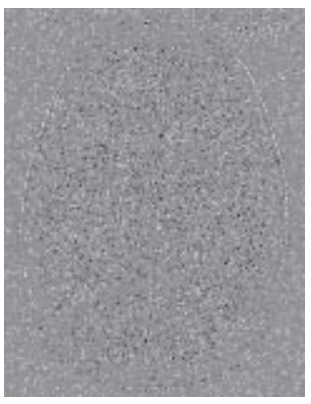

(e)

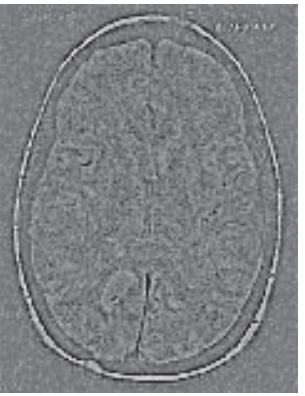

(b)

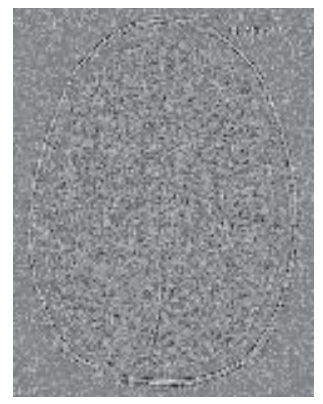

(f)

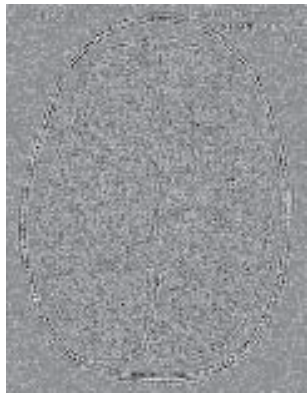

(c)

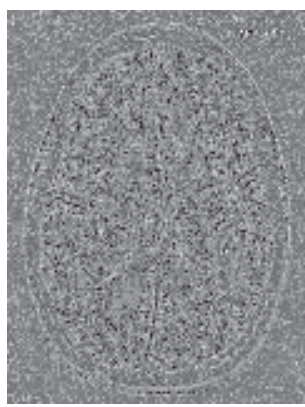

(g)

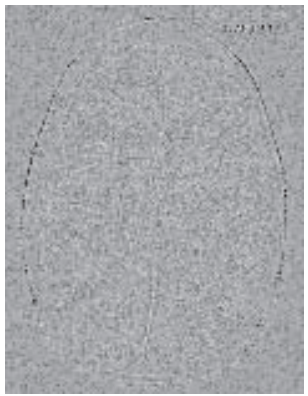

(d)

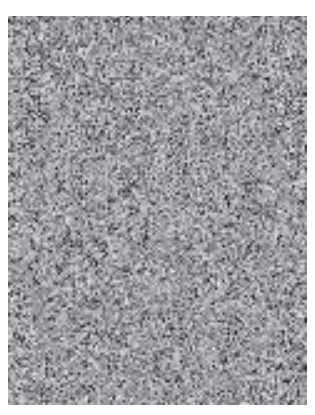

(h)

Fig. 9. Qualitative comparison of the method noise results obtained with the different filters for a transverse section of the head: (a) proposed method, (b) ADF, (c) wavelet, (d) median, (e) bilateral filter, (f) NL-mean, (g) TV, and (h) Gaussian noise (reference).

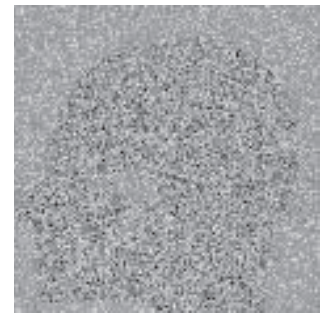

(a)

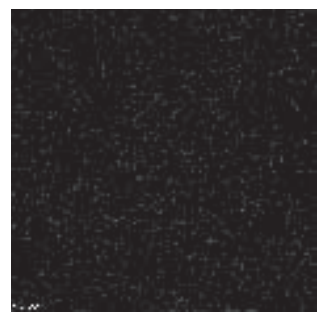

(e)

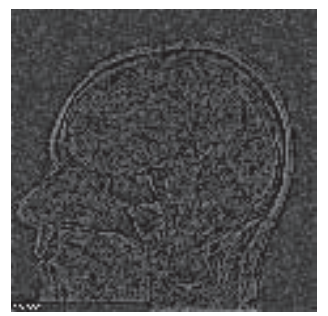

(b)

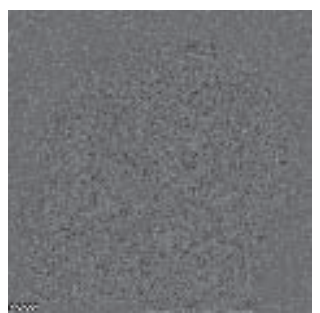

(f)

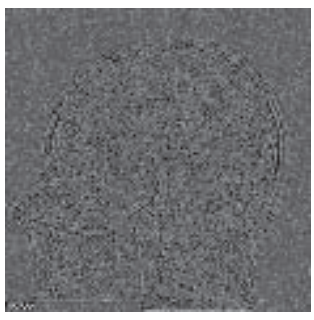

(c)

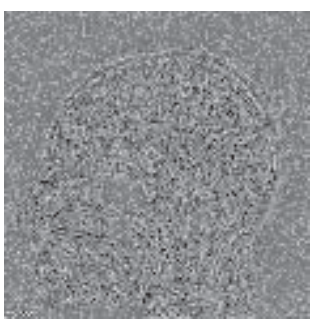

(g)

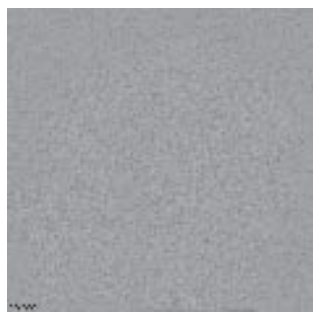

(d)

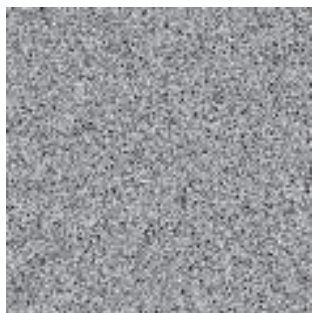

(h)

Fig. 10. Qualitative comparison of the method noise results obtained with the different filters in a sagittal head section: (a) proposed method, (b) ADF, (c) wavelet, (d) median, (e) bilateral filter, (f) NL-mean, (g) TV, and (h) Gaussian noise (reference).

Table I. Image quality evaluation metrics at statistical measurements; for different filter types.

\begin{tabular}{lcccc}
\hline & \multicolumn{4}{c}{ Feature set } \\
\cline { 2 - 5 } Filter types & SNR & PSNR & MSE & RMSE \\
\hline Median & $\mathbf{2 3 . 8 1 5 7}$ & $\mathbf{3 3 . 7 1 0 1}$ & $\mathbf{8 . 5 3}$ & $\mathbf{2 . 9 2}$ \\
Anisotropic & $\mathbf{2 2 . 6 7 3 0}$ & $\mathbf{3 0 . 9 5 1 0}$ & 15.37 & 3.92 \\
Wavelet & 21.4370 & $\mathbf{2 7 . 3 0 6 1}$ & 16.81 & 4.10 \\
TV & $\mathbf{2 4 . 7 7 6 7}$ & $\mathbf{3 0 . 6 3 5 3}$ & $\mathbf{1 4 . 3 8}$ & $\mathbf{3 . 7 6}$ \\
Bilateral & $\mathbf{2 3 . 2 9 2 1}$ & $\mathbf{2 8 . 0 4 8 2}$ & $\mathbf{1 1 . 7 1}$ & $\mathbf{3 . 4 2}$ \\
NLM & $\mathbf{2 5 . 5 0 3 0}$ & $\mathbf{3 5 . 3 9 2 8}$ & $\mathbf{5 . 7 6}$ & $\mathbf{2 . 4 0}$ \\
WDBF & $\mathbf{2 6 . 7 1 2 5}$ & $\mathbf{3 6 . 5 7 3 6}$ & $\mathbf{4 . 4 1}$ & $\mathbf{2 . 1 0}$ \\
\hline
\end{tabular}

Table II. Image quality evaluation metrics at statistical measurements; for different filter types.

\begin{tabular}{lcccc}
\hline & \multicolumn{4}{c}{ Feature set } \\
\cline { 2 - 5 } Filter types & SNR & PSNR & MSE & RMSE \\
\hline Median & $\mathbf{2 4 . 9 1}$ & $\mathbf{3 4 . 0 1}$ & $\mathbf{7 . 9 5}$ & $\mathbf{2 . 8 2}$ \\
Anisotropic & $\mathbf{2 1 . 4 3}$ & $\mathbf{3 0 . 0 4}$ & 16.97 & 4.12 \\
Wavelet & 19.76 & $\mathbf{2 6 . 8 3}$ & 17.89 & 4.23 \\
TV & $\mathbf{2 3 . 8 3}$ & $\mathbf{3 2 . 4 2}$ & $\mathbf{1 5 . 1 3}$ & $\mathbf{3 . 8 9}$ \\
Bilateral & $\mathbf{2 2 . 7 7}$ & $\mathbf{2 7 . 8 4}$ & $\mathbf{1 0 . 9 6}$ & $\mathbf{3 . 3 1}$ \\
NLM & $\mathbf{2 5 . 5 3}$ & $\mathbf{3 5 . 3 4}$ & $\mathbf{5 . 8 6}$ & $\mathbf{2 . 4 2}$ \\
WDBF & $\mathbf{2 6 . 6 0}$ & $\mathbf{3 5 . 9 6}$ & $\mathbf{5 . 3 8}$ & $\mathbf{2 . 3 2}$ \\
\hline
\end{tabular}




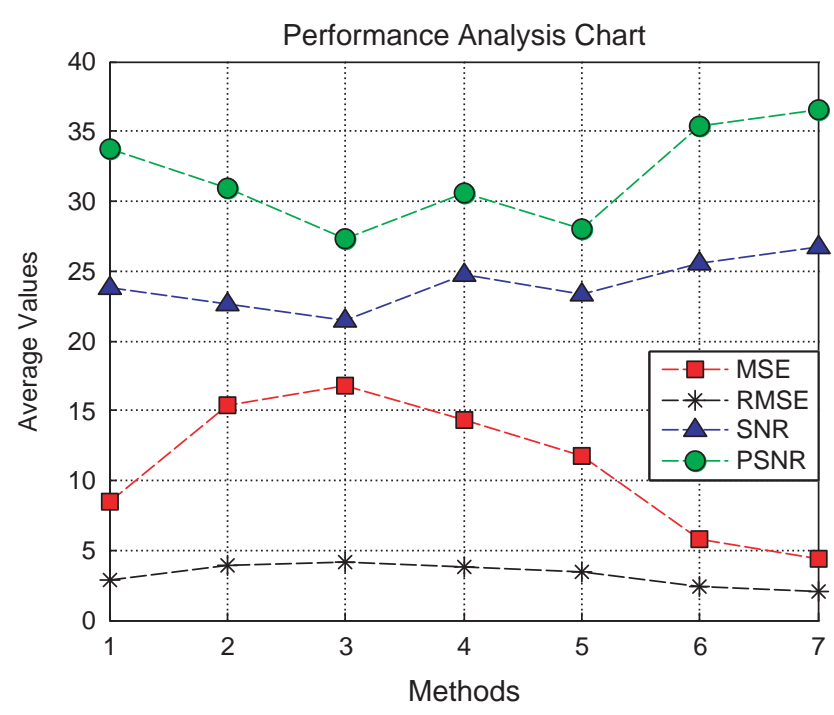

Fig. 11. Comparison of the image quality evaluation metrics for different filters (1: ADF, 2: wavelet filtering, 3: median, 4: bilateral filter, 5: NL-mean, 6: TV, and 7: proposed method). The proposed method outperforms the others in almost all the cases (in terms of MSE, RMSE, SNR and PSNR).

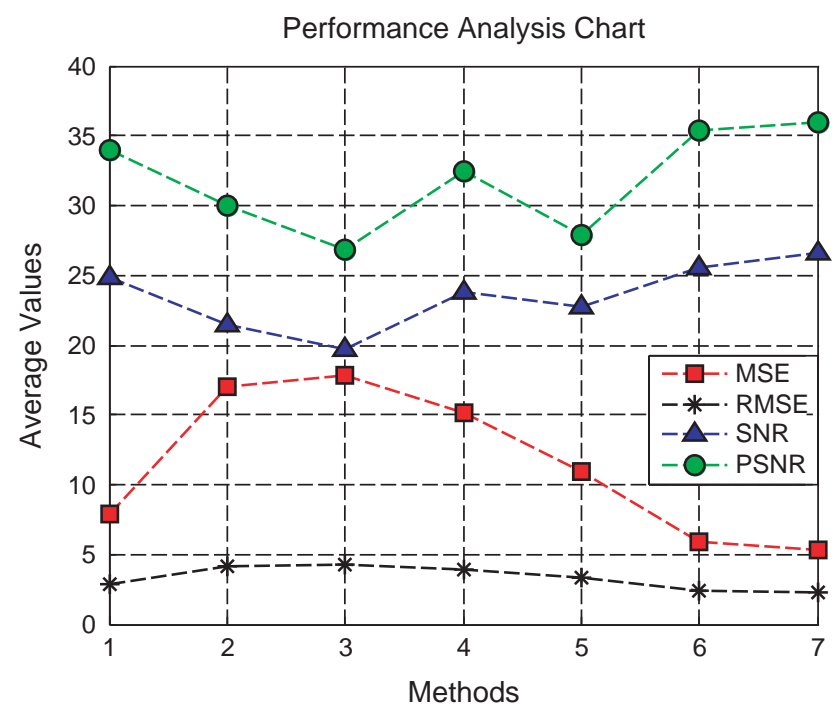

Fig. 12. Comparison of the image quality evaluation metrics for different filters (1: ADF, 2: wavelet filtering, 3: median, 4: bilateral filter, 5: NL-mean, 6: TV, and 7: proposed method). The proposed method outperforms the others in almost all the cases (in terms of MSE, RMSE, SNR and PSNR).

\section{CONCLUSIONS}

A denoising method for magnetic resonance images that combines bilateral filtering, wavelet decomposition and subband mixing is presented. The input image is decomposed into subbands using wavelet decomposition and different bilateral filtration parameters are used for each subband. The final image is formed by combining such subbands using subbands mixing. The performance of the new method is compared qualitatively and quantitatively to present techniques and shows promising results. The results suggest potential for practical application of the new method to boost SNR and hence reduce scan time in low magnetic field scanners.

Acknowledgment: Some of the images used in this work were obtained from the Biomedical Imaging Technology Center at the Georgia Tech/Emory University Biomedical Engineering.

\section{References and Notes}

1. A. Lukin, A multiresolution approach for improving quality of image denoising algorithms, Proc. Int. IEEE Conf. Acoustics, Speech, and Signal Processing (ICASSP'06) (2006), p. 857.

2. A. Buades, B. Coll, and J. Morel, A review of image denoising algorithms, with a new one. Multiscale Modeling and Simulation 4, 490 (2005).

3. C. Tomasi and R. Manduchi, Bilateral filtering for gray and color images, Proc. Int. Conf. Computer Vision (1998), p. 839.

4. M. Elad, On the origin of the bilateral filter and ways to improve it. IEEE Trans. Image Processing 11, 1141 (2002)

5. D. Barash, A fundamental relationship between bilateral filtering, adaptive smoothing, and the nonlinear diffusion equation. IEEE Trans. Pattern Analysis and Machine Intelligence 24, 844 (2002).

6. A. Buades, B. Coll, and J. Morel, Neighborhood filters and pde's. Numerische Mathematik 105, 1 (2006).

7. A. Macovski, Noise in MRI. Magn. Reson. Med. 36, 494 (1996).

8. W. A. Edelstein, G. Glover, C. Hardy, and R. Redington, The intrinistic signalto-noise ratio in NMR imaging. Magn. Reson. Med. 3, 604 (1986).

9. A. Papoulis, Random Variables, and Stochastic Processes, McGraw-Hill, New York (1984).

10. H. Gudbjartsson, and S. Patz, The Rician distribution of noisy MRI data. Magn. Reson. Med. 34, 910 (1995).

11. M. Zhang and B. K. Gunturk, Multiresolution bilateral filtering for image denoising. IEEE Trans. Image Process. 17, 2324 (2008).

12. B. A. Abrahim, Z. A. Mustafa, I. A. Yassine, N. Zayed, and Y. M. Kadah, Hybrid total variation and wavelet thresholding speckle reduction for medical ultrasound imaging. J. Med. Imaging Health Inf. 2, 1 (2012).

13. R. Gonzalez and R. Woods, Digital Image Processing, 2nd edn., PrenticeHall, New York (2002).

14. D. Sakrison, On the role of observer and a distortion measure in image transmission. IEEE Trans. Commun. 25, 1251 (1977).

15. P. Perona and J. Malik, Scale-space and edge detection using anisotropic diffusion. IEEE Trans. Pattern Analysis and Machine Intelligence 12, 629 (1990).

16. L. I. Rudin, S. Osher, and E. Fatemi, Nonlinear total variation based noise removal algorithms. Physica D 60, 259 (1992).

17. http://site.iugaza.edu.ps/amaghari/extra/course447/brain-tumor-detection/

18. http://www.melissamemorial.org/CMS/Show?id $=18$.

19. P. Coupé, P. Hellier, S. Prima, C. Kervrann, and C. Barillot, 3D wavelet subbands mixing for image denoising. Int. J. Biomed. Imag. 2008, 1 (2008). 